ENCYCLOPEEDE Encyclopédie berbère

BERBERE

$26 \mid 2004$

26 | Judaïsme - Kabylie

\title{
Kabylie : Littérature
}

D. Abrous

\section{(2) OpenEdition}

Journals

Édition électronique

URL : http://journals.openedition.org/encyclopedieberbere/1434

DOI : 10.4000/encyclopedieberbere.1434

ISSN : 2262-7197

\section{Éditeur}

Peeters Publishers

\section{Édition imprimée}

Date de publication : 1 mai 2004

Pagination : 4071-4074

ISBN : 2-7449-0452-X

ISSN : 1015-7344

\section{Référence électronique}

D. Abrous, «Kabylie : Littérature », Encyclopédie berbère [En ligne], 26 | 2004, document K17, mis en ligne le 01 juin 2011, consulté le 14 décembre 2020. URL : http://journals.openedition.org/ encyclopedieberbere/1434; DOI : https://doi.org/10.4000/encyclopedieberbere.1434

Ce document a été généré automatiquement le 14 décembre 2020.

(c) Tous droits réservés 


\section{Kabylie : Littérature}

\section{Abrous}

1 La littérature kabyle ancienne était une littérature essentiellement orale ; intimement liée à la vie sociale, elle se ramifiait en plusieurs genres : la poésie, le conte, les chants de travail (chants des travaux agricoles, chants de la meule...), chants rituels, proverbes, devinettes, comptines...

2 Parmi ces genres d'inégale importance, la première place revenait à la poésie. Une part non négligeable de cette production poétique était villageoise et anonyme mais cette poésie pouvait aussi être l'œuvre de poètes reconnus (afșiḥ, ifșiḥen) comme Youcef ou Kaci (Yusef u Qasi). Outre ces poètes créateurs, existaient des poètes transmetteurs (ameddah, imeddahen); ceux-ci faisaient circuler des répertoires qu'ils avaient mémorisés de village en village, de tribu en tribu. Avant la conquête française, ces poètes quel que soit leur statut étaient des poètes itinérants.

3 À l'intérieur de ce genre dominant qu'était la poésie, on pouvait distinguer deux souschamps :

- La poésie religieuse comprenait un répertoire probablement très ancien de longs poèmes (taqsiț, tiqsiḍin) évoquant des personnages bibliques (Abraham, Moïse, Joseph, Job...) et de l'Islam (le prophète Mohamed mais aussi et surtout Ali) - ( $C f$. Mammeri, 1980). À ce répertoire, s'ajoutait une veine de poèmes édifiants (ddker, de l'arabe dikr: "évocation du nom de Dieu »). Ces longs poèmes hagiographiques sont encore vivants dans la mémoire en particulier dans les milieux religieux ; quant aux poèmes édifiants, ils constituent une veine très productive, alimentée par des producteurs le plus souvent anonymes. Dans certains villages, ces poèmes sont encore régulièrement chantés par des chœurs féminins et masculins lors des veillées funèbres.

- La poésie profane présentait une thématique très diversifiée : référence au code de l'honneur, aux valeurs guerrières, poésie satirique, gnomique ; la poésie lyrique, bien qu'elle ait constitué une veine très productive, n'accédait que difficilement à l'espace public.

4 La conquête française et les profonds bouleversements qu'elle entraîna (violence de la conquête militaire, déstructuration du tissu tribal, important mouvement migratoire vers les villes algériennes et vers la France) eurent des incidences très nettes sur le 
champ de la littérature: certains genres, sans avoir totalement disparu, se sont essoufflés; c'est le cas du conte, des chants rituels, proverbes, comptines. Ces genres, bien qu'ils soient encore vivants, sont aujourd'hui très peu productifs. Dans ces profonds bouleversements qui pourraient s'apparenter à une véritable sélection par l'Histoire, seule la poésie a réellement survécu; elle a réussi à traduire aussi bien la violence $\mathrm{du}$ choc colonial que les profonds bouleversements qui s'ensuivirent. Les Poésies populaires de la Kabylie du Jurjura collectées par Hanoteau (1867) sont une véritable chronique de la conquête vue par les Kabyles; quant à Si Mohand, le poète errant, il rendit compte avec fidélité de la période qui fut perçue par les Kabyles comme la fin d'un monde.

5 Enfin, une des conséquences indirectes de la conquête française fut l'appropriation de l'écrit par les élites autochtones formées à l'école moderne ; cette appropriation donna naissance à une littérature écrite. L'émergence de cette littérature fut un processus long et complexe : entre la Méthode de langue kabyle de Saïd Boulifa en 1913 et le premier roman - Asfel de Rachid Aliche - paru en 1981, près de 70 ans se sont écoulés. Les premiers instituteurs, comme Boulifa, Ben Sedira, Cid Kaoui avaient produit à l'écrit des textes ethnographiques; ils avaient aussi fixé des textes de littérature orale (ce fut le cas des poèmes de Si Mohand collectés et publiés par Boulifa), mais ils n'avaient pas produit de textes littéraires.

6 Le premier auteur de textes littéraires écrits fut Bélaïd Aït Ali; celui-ci, mort prématurément à 39 ans en 1950, fut l'auteur d'un seul ouvrage que le Fichier de Documentation Berbère (FDB) publia en 1962 sous le titre : Les cahiers de Bélaïd ou la Kabylie d'antan. Cet ouvrage est en réalité un recueil de poèmes (isefra), de contes (timucuha) et de "nouvelles» (amexlud). Les textes figurant sous la rubrique amexluḍ(mélanges) s'apparenteraient à ce que l'on pourrait appeler des scènes de la vie quotidienne en Kabylie, une version anticipée et écrite en kabyle de Jours de Kabylie de Mouloud Feraoun. Amexluḍsignifie "mélange » (un peu confus) d'éléments divers; la difficulté d'une dénomination précise rend bien compte du caractère nouveau de ce genre, c'est pourtant ce genre difficile à dénommer qui préfigure ce que seront les nouvelles formes de production littéraire écrite. Analysant cette situation de transition, Paulette Galand-Pernet écrira en 1973: "Ce que prouvent Boulifa et Bélaïd Aït Ali, c'est qu'une ceuvre de longue haleine est possible. Si l'on n'a encore vu paraître aucun roman en berbère, cela tient à des raisons économiques et sociales et non à un manque de moyens littéraires » (Galand-Pernet 1973 : 318).

7 C'est le début des années 1970 qui constitue un véritable tournant pour la littérature kabyle qu'elle soit orale ou écrite. La néo-chanson s'est imposée avec des noms comme Idir, Aït-Manguellet, Ferhat, Matoub Lounès, le groupe Djurdjura... Il s'agit de chansons à textes; à la différence des poètes traditionnels, les auteurs contemporains écrivent leurs poèmes et la langue de cette poésie moderne tout en réactivant des archaïsmes, des métaphores et des motifs anciens, puise à des degrés divers dans la néologie.

Pour la littérature écrite, la tendance qui consiste à produire dans la langue et non pas seulement à y fixer des textes oraux devient irréversible, étendant ainsi l'écrit à des domaines tout à fait nouveaux: littérature, production de lexiques spécialisés (mathématiques, informatique, linguistique...), traduction, presse. De tous ces champs investis par l'écrit moderne, le champ de la littérature est sans conteste le plus important. C'est la néo-littérature qui a servi et qui sert encore de laboratoire à cette langue en gestation. Dans ce champ littéraire complètement renouvelé depuis le début 
des années 1970, on notera la traduction d'œuvres algériennes (Kateb, Feraoun), étrangères (Brecht, Beckett, Molière), la naissance de genres littéraires nouveaux tels que le théâtre, la nouvelle, le roman.

9 Le théâtre est lié au nom de Mohand-ou-Yahia qui fit œuvre de pionnier à partir du début des années 1970 par ses traductions-adaptations d'œuvres de Brecht, Kateb, Molière, etc. Avec la chanson le théâtre constitue un véritable pont entre l'écriture et l'oralité.

10 À la même période, en 1981, Asfel de Rachid Aliche annonce la naissance du genre romanesque.

11 C'est dans la littérature écrite que la langue est investie comme un véritable laboratoire. Cette langue, dans laquelle s'expriment de profondes fractures et une ultime lutte pour la survie, est en même temps malmenée et jalousement préservée. Cette langue est en effet traversée par une profonde dynamique qui touche d'abord le lexique par l'intégration importante de néologismes et à un degré moindre la syntaxe : l'interférence avec la syntaxe du français constitue une tendance lourde pour le kabyle écrit en général; sans échapper totalement à cette tendance, la langue de la néolittérature est relativement épargnée quant à la prégnance des calques syntaxiques.

12 Sur le plan du contenu, un thème majeur traverse comme une lame de fond l'ensemble de cette néo-littérature qu'elle soit orale ou écrite : il s'agit de la contestation politique et de la quête identitaire. La poésie de ces trente dernières années, aussi bien que la production romanesque porte une "charge » de contestation d'une rare virulence à l'égard de l'État. La thématique de la contestation n'est pas née ex nihilo: le thème de la résistance a toujours été fortement présent dans la poésie kabyle ( $C f$. Benbrahim 1982 ; Chaker 1989); le lien entre poésie et résistance était si nettement perçu pendant la période coloniale que la circulation des poètes était très sévèrement surveillée après l'insurrection de 1871. La fronde contestataire des poètes contemporains est dirigée contre l'État algérien en raison du déni identitaire. C'est dans ce contexte que la quête identitaire occupe une place prépondérante: cette quête, rendue par des moyens différents, est omniprésente dans les romans de Rachid Aliche, de Saïd Sadi, de Amar Mezdad; elle peut revêtir dans un roman comme Asfel (R. Aliche, 1981) des formes pathétiques.

13 Un autre thème lié aux deux précédents sous-tend en particulier la production romanesque, il s'agit du thème de l'éclatement : à l'image de la culture à laquelle ils appartiennent, les héros de ces romans sont des hommes en crise dans une situation de crise. Cet éclatement, symbolisé dans les romans d'Aliche par l'image de l'amphore brisée, peut mener les héros jusqu'au suicide. Dans le roman d'Amar Mezdad, id d wass (Mezdad 1990), c'est la frêle silhouette de la vieille mère qui assure le lien entre hier et aujourd'hui, le village et la ville, c'est elle qui assure la permanence entre ces profondes fractures, évitant ainsi l'éclatement.

14 Cette thématique, bien que très actuelle, prend solidement ancrage dans la symbolique berbère. C'est cette capacité de se projeter dans l'avenir sans se déraciner qui fait l'originalité de la littérature kabyle aujourd'hui. 


\section{BIBLIOGRAPHIE}

AïT ALI (Belaïd), 1962, « Les cahiers de Bélaïd ou la Kabylie d'antan » in Fichier de Documentation

Berbère (FDB).

ALICHE R., 1981, Asfel (roman), Mussidan, Lyon.

ALICHE R., 1986, Faffa (roman), Mussidan, Lyon.

BASSET H., 1920, Essai sur la littérature des Berbères, Carbonnel, Alger.

BENBRAHIM-BENHAMADOUCHE M., 1982, La poésie populaire kabyle et la résistance à la colonisation de 1830 à 1962, Paris, École Des Hautes Études en Sciences Sociales (EHESS); Thèse de Doctorat sous la direction de Camille Lacoste-Dujardin.

BOULIFA (Si Amar ou Saï), 1913, Méthode de langue kabyle (2e année) étude linguistique et sociologique sur la Kabylie du Djurdjura, Jourdan, Alger.

BOUNFOUR A., 1999, Introduction à la littérature berbère I : la poésie, Peeters, Paris - Louvain.

CHAKER S., 1982, «Structures formelles de la poésie kabyle » in Actes de la table ronde «Littérature orale », CRAPE - OPU, Alger.

CHAKER S., 1987, « Documents sur les précurseurs. Deux instituteurs kabyles : Si Amar ou Saïd Boulifa et M. A Lechani » in RoMm, 44, p. 97-115. Édisud, Aix-en-Provence (sous la direction de Salem Chaker).

CHAKER S., 1989, « Une tradition de résistance et de lutte : la poésie berbère kabyle. Un parcours poétique » in RMMM, 51, p :11-31. Édisud, Aix-en-Provence (sous la direction de Salem Chaker). CHAKER S., 2001, « BOULIFA Si Amar ou Saïd (1865-1931) : le grand précurseur berbé-risant » in Hommes et Femmes de Kabylie, p : 119-123. Édisud, Aix-en-Provence Ina-yas, Alger.

GALAND-PERNET P., 1973, « Tradition et modernité dans les littératures berbères » in Actes du Premier Colloque des Cultures Méditerranéennes d'Influence Arabo-Berbère, SNED, Alger.

GALAND-PERNET P., 1998, Littératures berbères. Des voix. Des lettres, Presses Universitaires de France (PUf), Paris.

HANOTEAU A., 1867, Poésies populaires de la Kabylie du Jurjura, Imprimerie Impériale, Paris.

LACOSTE-DUJARDin C, 1970, Le conte kabyle. Étude ethnologique. Maspéro, Paris.

MAMMERI M., 1969, Les Isefra, poèmes de Si Mohand ou Mhand, Maspéro, Paris.

MAMMERI M., 1980, Poémes kabyles anciens, Maspéro, Paris.

MAMMERI M., 1990, Inna-yas Ccix Muhend, Cheikh Mohand a dit, édition Inna-yas, Alger.

MEROLLA D., 1997, Gender and Community $i$ the kabyle literary space. Research School, cNwS, Leiden. MEZDAD A., 1990, Iọ d wass (roman), édition Asalu - Azar, Alger.

MEZDAD A., 2000, Tagrest urүu (roman), édition Ayamun, Béjaïa.

SADI S., 1983, Askuti (roman), Imedyazen, Paris.

yaCine-TitouH T., 1988, L'izli ou l'amour chanté en kabyle, Maison des Sciences de l'Homme (MSH), Paris. 
YACINE-Titoun T., 1989, Aït-Manguellat chante, La Découverte - Awal, Paris.

ZENIA S., 1995, Tafrara Aurore (roman), L'Harmattan - Awal, Paris.

INDEX

Mots-clés : Littérature, Kabylie 